Supporting Information for

\title{
Novel Donor-Electret-Acceptor Framework for Higher Charge Transfer and Distance of Charge Transfer through Dipole Engineering
}

Talapunur Vikramaditya and Shiang-Tai Lin*

Computational Molecular Engineering Laboratory, Department of Chemical Engineering, National Taiwan University, Taipei 10617, Taiwan.

*email: stlin@ntu.edu.tw

Table S.1. Oscillator strengths corresponding to the first excited states for the DBA molecules shown in scheme.2. Range separation parameters with *tuning approach are shown in parenthesis.

\begin{tabular}{|l|c|c|c|}
\hline$D B_{n} A$ & B3LYP & M062X & LC-wHPBE* \\
\hline $\mathbf{1}$ & 0.6260 & 0.6701 & $0.6516(0.28)$ \\
\hline $\mathbf{2}$ & 0.6363 & 0.8922 & $0.8824(0.25)$ \\
\hline $\mathbf{3}$ & 0.4591 & 1.1837 & $1.2095^{* *}(0.24)$ \\
\hline
\end{tabular}

Note: In most of the cases $1^{\text {st }}$ exited state exhibit appreciable oscillator strengths as shown. ${ }^{* *} 2^{\text {nd }}$ excited state of $\mathrm{DB}_{3} \mathrm{~A}$ exhibit optical gap of $3.85 \mathrm{eV}$ with oscillator strength 1.2095 and $1^{\text {st }}$ excited state with an optical gap of $3.77 \mathrm{eV}$ and oscillator strength of 0.1614 . Due to the close proximity of $1^{\text {st }}$ and $2^{\text {nd }}$ excited states and the weak oscillator strength of $1^{\text {st }}$ excited state it can be hard to detect experimentally. Since the comparison is against the experimental $\lambda_{\max }$, we compare with the $2^{\text {nd }}$ excited state owing to the close proximity with the $1^{\text {st }}$ excited state and higher oscillator strength.

Table S.2. $0-0$ transition energies (eV) evaluated by different theoretical approaches. * corresponds to tuning approach.

\begin{tabular}{|l|c|c|c|}
\hline $\mathbf{D B}_{n} \mathbf{A}$ & B3LYP & M062X & LC-wHPBE* \\
\hline $\mathbf{1}$ & 2.77 & 3.15 & 3.09 \\
\hline $\mathbf{2}$ & 1.97 & 2.95 & 2.95 \\
\hline $\mathbf{3}$ & 1.84 & 2.93 & 2.92 \\
\hline
\end{tabular}

\section{Optical Gaps and Oscillator strengths}

Incident photon to-current conversion efficiency (IPCE) and LHEs are expressed as

$$
I P C E=L H E(\lambda) * \Phi_{i n j} * \eta_{r e g} * \eta_{c c}-(1)
$$




$$
\operatorname{LHE}(\lambda)=1-10^{-f}-(2)
$$

$\Phi_{\text {inj }}, \eta_{\text {reg }}, \eta_{\text {cc }}$ and $f$ are the quantum yield of charge injection and the dye regeneration and charge collection efficiencies and oscillator strengths respectively.

Optical gaps and oscillator strengths of Donor-Bridge ${ }_{n}$-Acceptor and Donor-Electret ${ }_{n}$ Acceptor $\left(D E_{n} A\right) ;\left(D B_{n} A\right) ; n=2-9$ are given.

Table: S.3

\begin{tabular}{|c|c|c|}
\hline $\begin{array}{c}\text { Cyclopentadiene- } \\
\text { DB }_{\mathbf{n}} \mathbf{A}\end{array}$ & OPT GAP (eV) & OS \\
\hline 2 & 2.5261 & 0.7607 \\
\hline 3 & 2.2751 & 1.0196 \\
\hline 4 & 2.135 & 1.3075 \\
\hline 5 & 2.1071 & 1.6153 \\
\hline 6 & 2.0375 & 1.9444 \\
\hline 7 & 2.0199 & 2.2876 \\
\hline 8 & 1.9944 & 2.6334 \\
\hline 9 & 1.9827 & 2.9758 \\
\hline
\end{tabular}

Table: S.4

\begin{tabular}{|c|c|c|}
\hline $\begin{array}{c}\text { Cyclopentadiene- } \\
\mathrm{DE}_{\mathrm{n}} \mathbf{A}\end{array}$ & OPT GAP (eV) & OS \\
\hline 2 & 2.2124 & 0.6517 \\
\hline 3 & 1.8708 & 0.8303 \\
\hline 4 & 1.8387 & 1.0315 \\
\hline 5 & 1.7689 & 1.2421 \\
\hline 6 & 1.7745 & 1.5123 \\
\hline 7 & 1.7234 & 1.7687 \\
\hline 8 & 1.7291 & 2.1007 \\
\hline 9 & 1.7312 & 2.4522 \\
\hline
\end{tabular}

Table: S.5

\begin{tabular}{|c|c|c|}
\hline Pyrrole-DB $_{\mathbf{n}} \mathbf{A}$ & OPT GAP (eV) & OS \\
\hline 2 & 3.3471 & 0.6705 \\
\hline 3 & 3.3847 & 0.8346 \\
\hline 4 & 3.4011 & 0.9933 \\
\hline 5 & 3.3585 & 1.1812 \\
\hline 6 & 3.3686 & 1.2557 \\
\hline 7 & 3.3617 & 1.3124 \\
\hline 8 & 3.3762 & 1.3273 \\
\hline 9 & 3.3818 & 1.3458 \\
\hline
\end{tabular}


Table: S.6

\begin{tabular}{|c|c|c|}
\hline Pyrrole-DE ${ }_{n} A$ & OPT GAP (eV) & OS \\
\hline 2 & 3.0889 & 0.7301 \\
\hline 3 & 2.7983 & 0.9744 \\
\hline 4 & 2.6747 & 1.1682 \\
\hline 5 & 2.6514 & 1.363 \\
\hline 6 & 2.6506 & 1.5397 \\
\hline 7 & 2.6528 & 1.6929 \\
\hline 8 & 2.6568 & 1.8154 \\
\hline 9 & 2.6587 & 1.9143 \\
\hline
\end{tabular}

Table: S.7

\begin{tabular}{|c|c|c|}
\hline Furan-DB $\mathbf{~}_{\mathbf{n}} \mathbf{A}$ & OPT GAP & OS \\
\hline 2 & 3.1547 & 0.7289 \\
\hline 3 & 3.0728 & 1.036 \\
\hline 4 & 3.0769 & 1.4186 \\
\hline 5 & 3.0393 & 1.8179 \\
\hline 6 & 3.0344 & 2.2436 \\
\hline 7 & 3.0258 & 2.6679 \\
\hline 8 & 3.0168 & 3.0879 \\
\hline 9 & 3.0084 & 3.507 \\
\hline
\end{tabular}

Table: S.8

\begin{tabular}{|c|c|c|}
\hline Furan- $\mathrm{DE}_{\mathrm{n}} \mathrm{A}$ & OPT GAP & OS \\
\hline 2 & 3.148 & 0.6363 \\
\hline 3 & 2.7116 & 0.9016 \\
\hline 4 & 2.6177 & 1.1543 \\
\hline 5 & 2.5968 & 1.4445 \\
\hline 6 & 2.5899 & 1.7571 \\
\hline 7 & 2.586 & 2.079 \\
\hline 8 & 2.5823 & 2.399 \\
\hline 9 & 2.5773 & 2.7443 \\
\hline
\end{tabular}


Table: S.9

\begin{tabular}{|c|c|c|}
\hline $\begin{array}{l}\text { Thiophene- } \\
\text { DB } \mathbf{n}_{\mathbf{A}} \mathbf{A}\end{array}$ & OPT GAP & OS \\
\hline 2 & 3.0332 & 0.7545 \\
\hline 3 & 2.9873 & 1.0742 \\
\hline 4 & 3.0411 & 1.5009 \\
\hline 5 & 3.0779 & 1.9593 \\
\hline 6 & 3.1333 & 2.4317 \\
\hline 7 & 3.042 & 2.9052 \\
\hline 8 & 3.0782 & 3.3585 \\
\hline 9 & 3.0636 & 3.8119 \\
\hline
\end{tabular}

Table: S.10

\begin{tabular}{|c|c|c|}
\hline $\begin{array}{c}\text { Thiophene- } \\
\mathbf{D E}_{\mathrm{n}} \mathbf{A}\end{array}$ & OPT GAP & OS \\
\hline 2 & 2.9187 & 0.6244 \\
\hline 3 & 2.6308 & 0.9541 \\
\hline 4 & 2.4699 & 1.2764 \\
\hline 5 & 2.4202 & 1.6326 \\
\hline 6 & 2.3494 & 1.9838 \\
\hline 7 & 2.3346 & 2.3831 \\
\hline 8 & 2.3233 & 2.7986 \\
\hline 9 & 2.3144 & 3.2224 \\
\hline
\end{tabular}

Table S.11. Tuned range separation parameters $\left(\omega=\right.$ bohr $\left.{ }^{-1}\right)$, charge transfer $\left(q_{\mathrm{CT}}\right)$ and distance of charge transfer $\left(D_{C T}\right)$ are provided for donor and acceptor electrets $\left(E_{D}\right)_{n}=1$ 9 and $\left(E_{A}\right)_{n}=1-9$ respectively.

\begin{tabular}{|c|c|c|c|c|c|c|c|}
\hline $\begin{array}{c}\text { No. of } \\
\text { units } \\
E_{D}\end{array}$ & $\begin{array}{c}\text { Tuned } \\
\text { " } \omega \text { " }\end{array}$ & $q_{C T}(e)$ & $\begin{array}{l}D_{\mathrm{CT}} \\
(\AA)\end{array}$ & $\begin{array}{c}\text { No. of } \\
\text { units } \\
E_{A}\end{array}$ & $\begin{array}{l}\text { Tuned } \\
\text { " } \omega \text { " }\end{array}$ & $\mathbf{q}_{\mathrm{ct}}(\mathrm{e})$ & $\begin{array}{l}\mathrm{D}_{\mathrm{CT}} \\
(\AA)\end{array}$ \\
\hline 1 & 0.28 & 0.494 & 1.205 & 1 & 0.29 & 0.703 & 0.447 \\
\hline 2 & 0.22 & 0.416 & 1.277 & 2 & 0.23 & 0.534 & 1.271 \\
\hline 3 & 0.19 & 0.412 & 1.842 & 3 & 0.18 & 0.427 & 0.508 \\
\hline 4 & 0.17 & 0.417 & 2.351 & 4 & 0.18 & 0.404 & 0.419 \\
\hline 5 & 0.16 & 0.420 & 2.594 & 5 & 0.16 & 0.390 & 0.377 \\
\hline 6 & 0.15 & 0.421 & 2.739 & 6 & 0.15 & 0.385 & 0.219 \\
\hline 7 & 0.14 & 0.422 & 2.830 & 7 & 0.14 & 0.381 & 0.092 \\
\hline 8 & 0.14 & 0.419 & 2.370 & 8 & 0.14 & 0.383 & 0.223 \\
\hline 9 & 0.14 & 0.418 & 1.836 & 9 & 0.14 & 0.384 & 0.472 \\
\hline
\end{tabular}


Table S.12. Ground state (GS) Dipole moment (in Debye), Charge transfer qCT (e) and Distance of charge transfer $D_{\text {ст }}(\AA)$ for different DBA and DEDA systems shown in scheme 3 are given. Tuned range separation parameter is provided in parenthesis.

\begin{tabular}{|c|c|c|c|c|c|c|}
\hline & D & A & $E$ & GS-Dipole & $\mathbf{q}_{\mathrm{CT}}$ & $D_{C T}$ \\
\hline DBA (0.20) & Diamine & Fluoro & - & 5.00 & 0.43 & 2.15 \\
\hline$D_{D} A(0.20)$ & Diamine & Fluoro & Hydroxyl & 4.20 & 0.51 & 3.07 \\
\hline DBA (0.19) & Diamine & Cyano & - & 10.81 & 0.50 & 3.55 \\
\hline$D E_{D} A(0.20)$ & Diamine & Cyano & Hydroxyl & 2.27 & 0.62 & 4.40 \\
\hline DBA (0.18) & Diamine & Cyanoacryl & - & 11.69 & 0.55 & 4.43 \\
\hline$D E_{D} A(0.18)$ & Diamine & Cyanoacryl & Methoxy & 4.84 & 0.67 & 4.83 \\
\hline DBA $(0.20)$ & Methoxy & Cyano & - & 7.95 & 0.45 & 3.02 \\
\hline$D E_{D} A(0.20)$ & Methoxy & Cyano & Hydroxyl & 2.37 & 0.55 & 3.88 \\
\hline
\end{tabular}




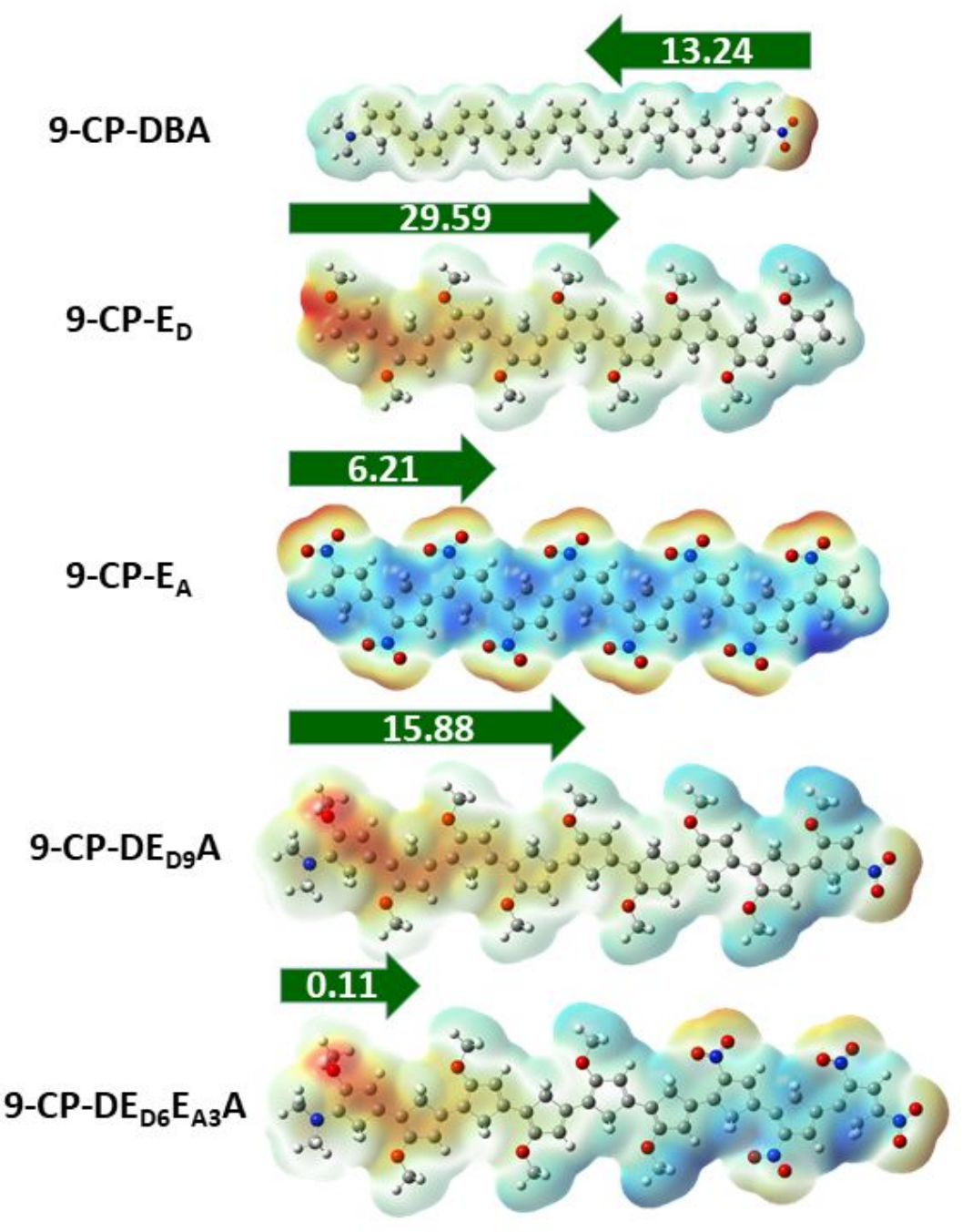

Figure S.1. Electron density (isovalue $=0.0004$ ) mapped with electrostatic potential (color scheme: red for negative surface map values and blue for the positive ones). Dipole moment and direction along the bridge axis for 9-CP-DBA, Donor Electret 9-CP- $\mathrm{E}_{\mathrm{D}}$, Acceptor Electret 9-CP- $\mathrm{E}_{\mathrm{A}}$ and DEA architectures $9 C P-D E_{D 9} A, 9 C P-D E_{D 6} E_{A 3} A$ in the ground state are shown. Note: $C P$ corresponds to cyclopentadiene. 


\subsection{8}

\section{9-CP-DE $\mathrm{Dg}_{\mathrm{D}} \mathrm{A}$}

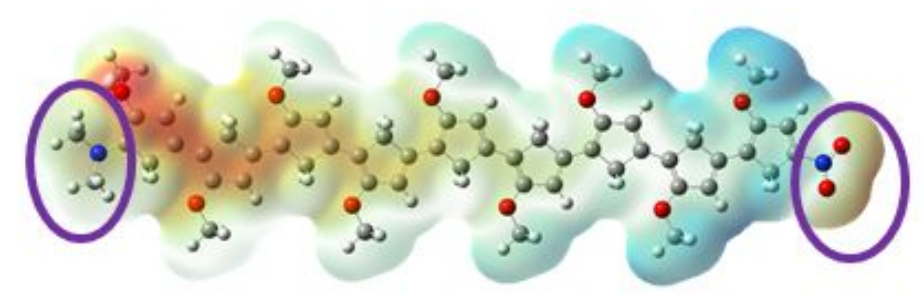

\subsection{3}

\section{9-CP-DE $\mathrm{Dg}_{\mathrm{A}} \mathrm{A}-\mathrm{rev}$}

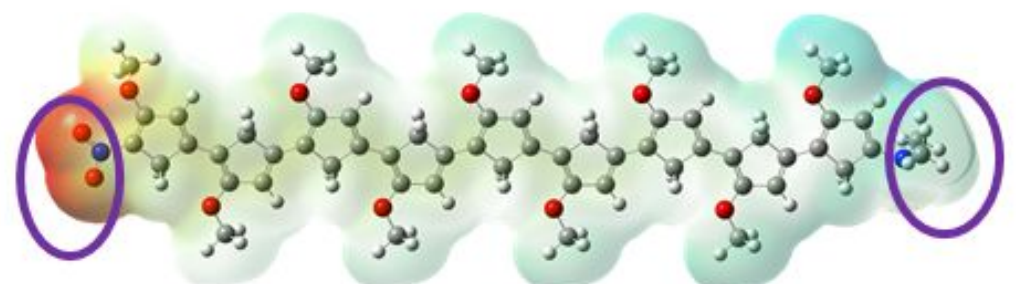

Figure S.2. Electron density (isovalue $=0.0004$ ) mapped with electrostatic potential (color scheme: red for negative surface map values and blue for the positive ones). Dipole moment and direction along the bridge axis for 9-CP-DE $A$. In 9-CP-DE $E_{D 9} A$ the donor electret $E_{D 9}$ dipole is oriented against the donor-acceptor duo at the terminal edges (Lower dipole moment). In the 9-CP-DE $E_{D 9} A-r e v$ the donor electret $\mathrm{E}_{\mathrm{D} g}$ dipole is oriented along the donor-acceptor duo at the terminal edges (Higher dipole moment). $C T$ and $\mathrm{D}_{\mathrm{CT}}$ of $9-\mathrm{CP}-\mathrm{DE}_{\mathrm{Dg}} \mathrm{A}$ and $9-\mathrm{CP}-\mathrm{DE} \mathrm{E}_{\mathrm{D} 9} \mathrm{~A}-\mathrm{rev}$ are $0.604 \mathrm{e}, 6.32 \AA$ and $0.428 \mathrm{e}$, $1.59 \AA ̊$ respectively.

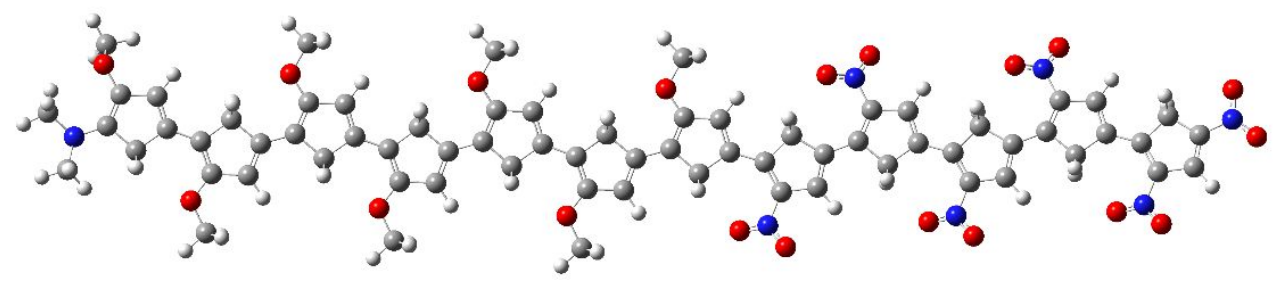

Figure S.3. Optimized molecular geometry of $12-C P-D E_{D 7} E_{A 5} A$ where $C P$ corresponds to cyclopentadiene, $E_{D}$ and $E_{A}$ corresponds to donor and acceptor electrets and $D$ and $A$ corresponds to terminal donor and acceptor groups. 


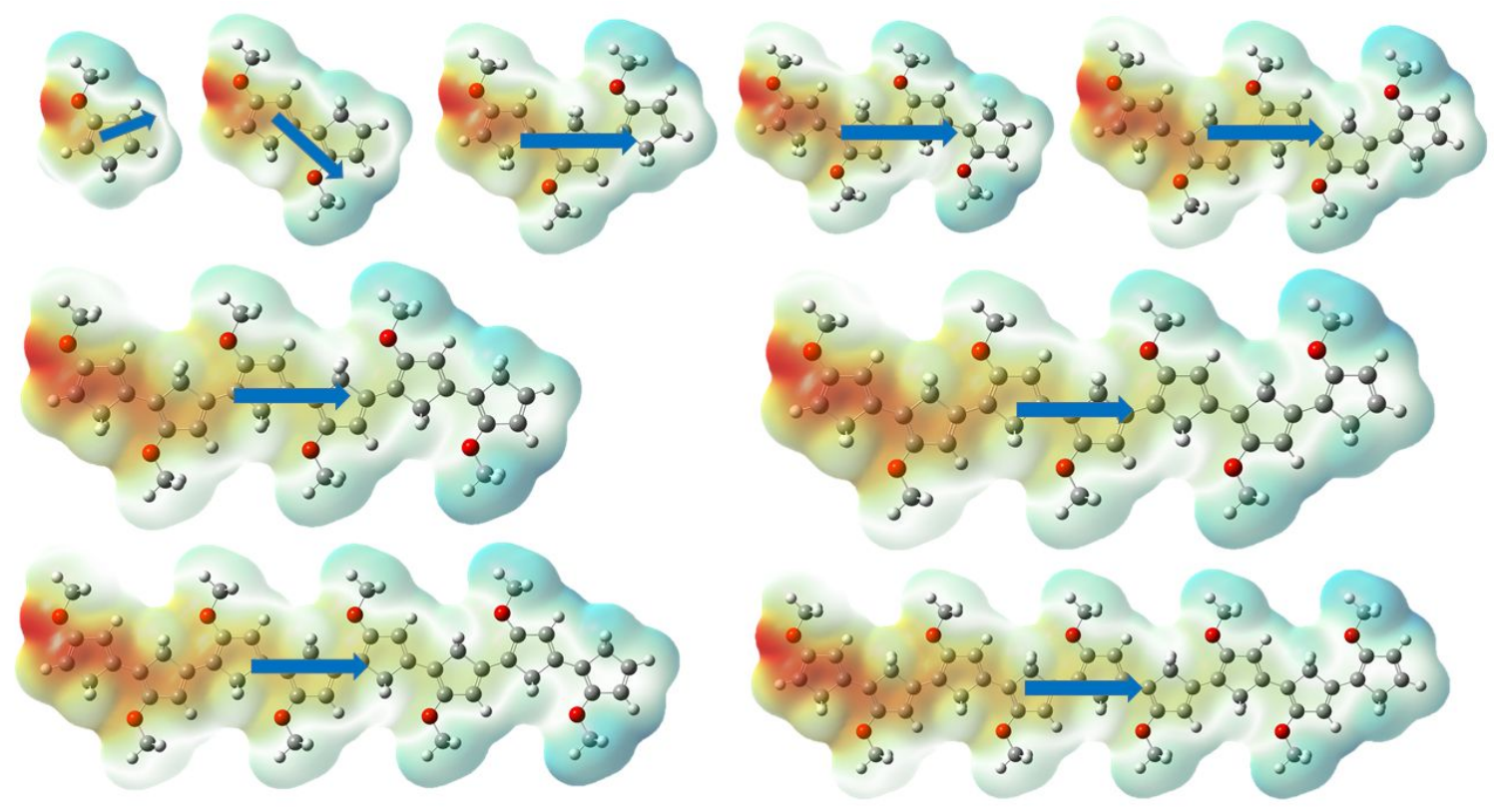

Figure S.4. Electron density (isovalue $=0.0004$ ) mapped with electrostatic potential (color scheme: red for negative surface map values and blue for the positive ones). Dipole moment projection along the bridge axis is shown for the methoxy donor electret $E_{D n}(n=1-9)$.

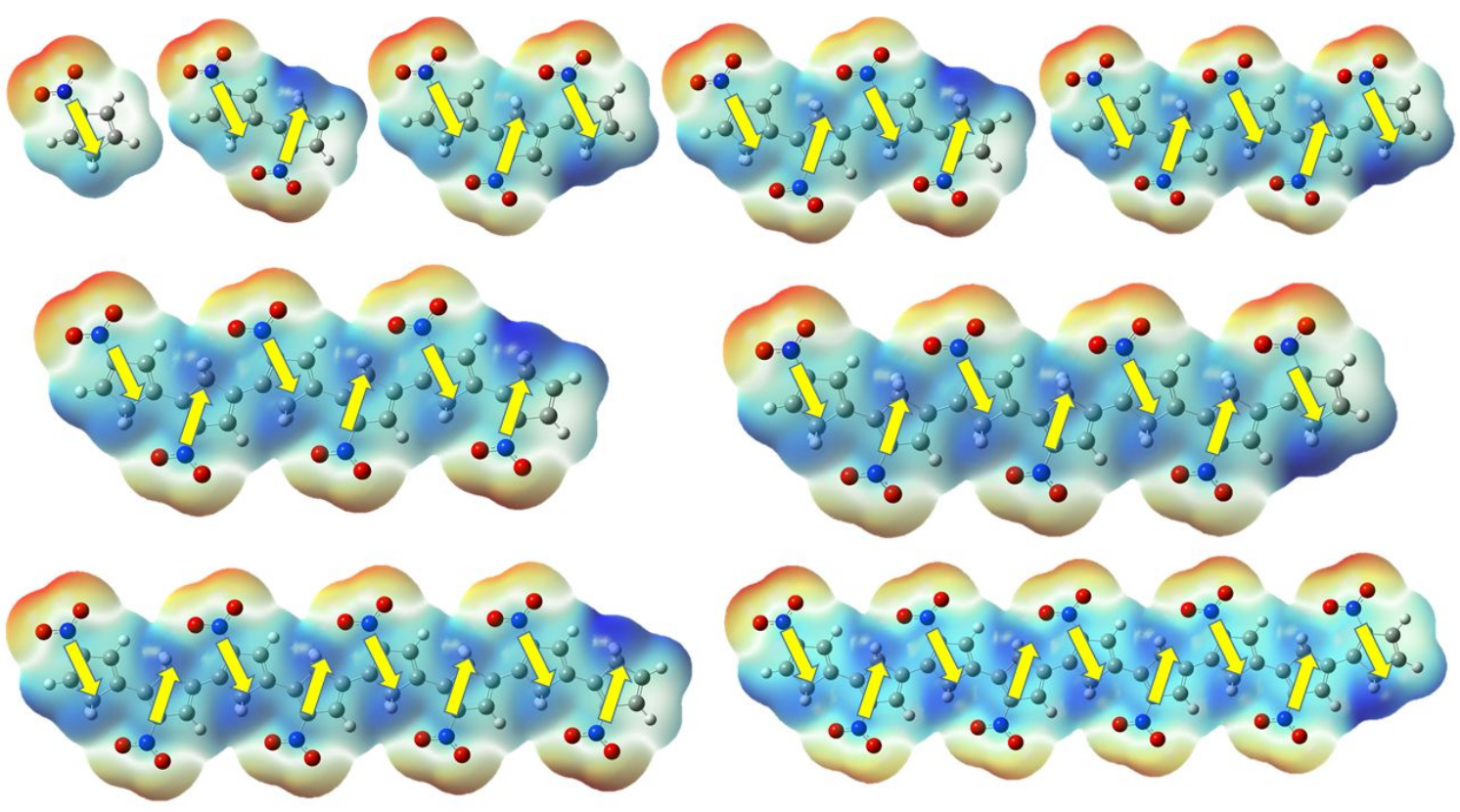

Figure S.5. Electron density (isovalue $=0.0004$ ) mapped with electrostatic potential (color scheme: red for negative surface map values and blue for the positive ones). Nitro groups along the bridge abstracts electrons from the bridge, the dipole components of individual bridge units are largely cancelled (especially with even bridges; $E_{A n}(n=1-9)$. 


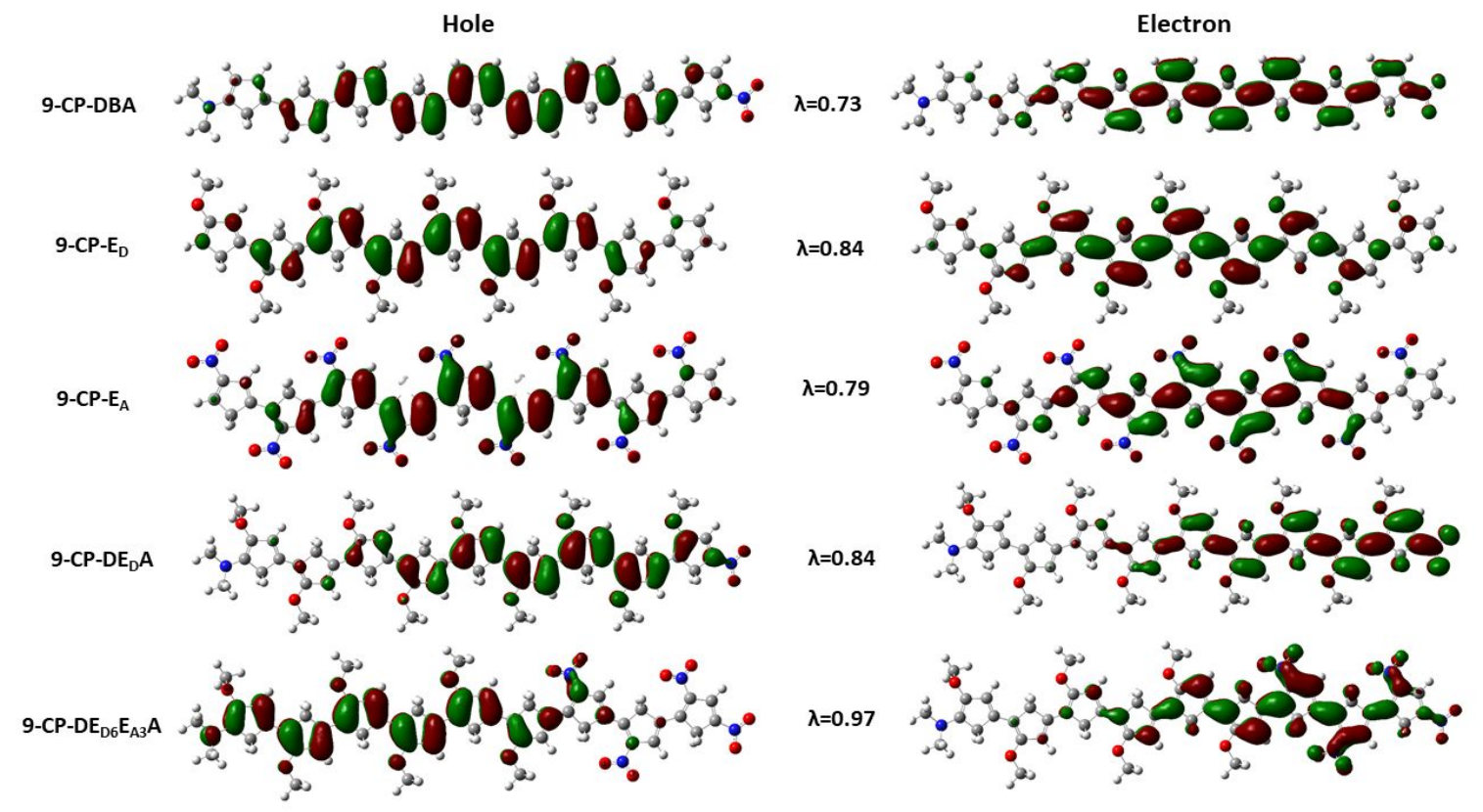

Figure S.6. Natural Transition orbital analysis corresponding to the first excited state. " $\lambda$ " corresponds to the excitation contribution on NTO pairs involved in the transition.

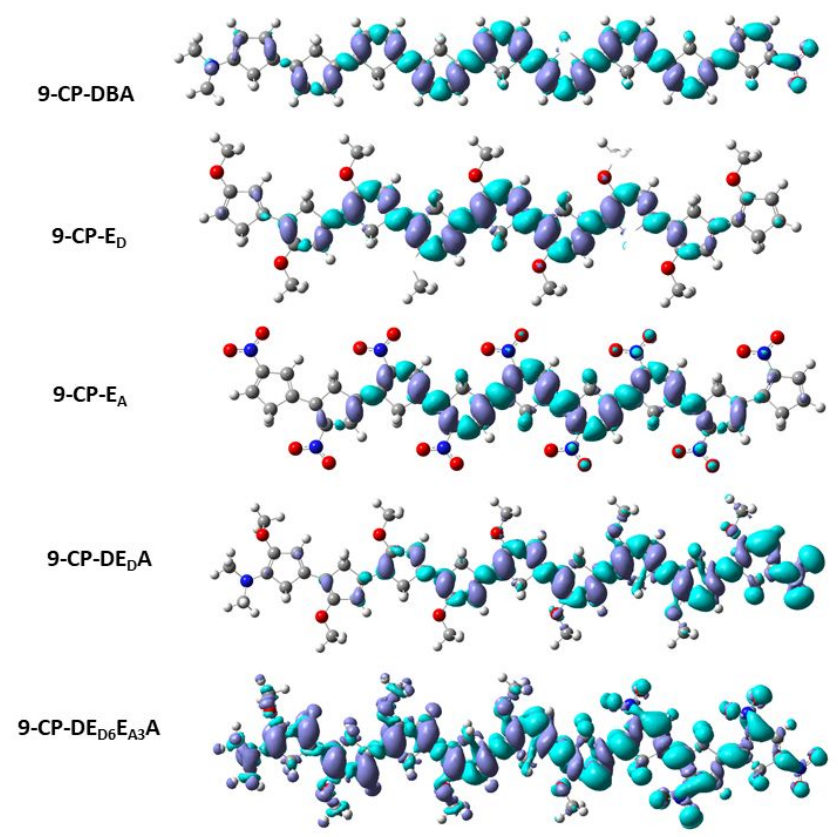

Figure S.7. Computed difference in total density for the ground and excited states $\Delta \rho_{E X}(r)-\Delta \rho_{G S}$ $(r)$. 